\title{
Magnetic resonance spectroscopy for assessment of brain injury in the rat model of sepsis
}

\author{
MIAOYUN WEN $^{1 *}$, ZHESI LIAN $^{2 *}$, LINQIANG HUANG $^{1}$, SENZHI ZHU $^{1}$, BEI HU $^{1}$, \\ YONGLI HAN $^{1}$, YIYU DENG ${ }^{1}$ and HONGKE ZENG ${ }^{3}$
}

\author{
${ }^{1}$ Department of Emergency and Critical Care Medicine, Guangdong General Hospital, Guangdong Academy \\ of Medical Sciences, Guangzhou, Guangdong 510080, P.R. China; ${ }^{2}$ Tufts University School of Medicine, \\ Boston, MA 02111, USA; ${ }^{3}$ Department of Acute Critical Care Medicine, Guangdong General Hospital, \\ Guangdong Academy of Medical Sciences, Guangzhou, Guangdong 510080, P.R. China
}

Received August 10, 2016; Accepted March 10, 2017

DOI: $10.3892 / \mathrm{etm} .2017 .5034$

\begin{abstract}
The diagnostic value of magnetic resonance spectroscopy (MRS), T2-weighted imaging (T2WI) and serum markers of brain injury in a rat model of sepsis were investigated. Rats were randomly divided into the control group and 6, 12 and $24 \mathrm{~h}$ after lipopolysaccharide-injection groups. Brain morphology and metabolism were assessed with T2WI magnetic resonance imaging (MRI) and MRS. Serum and brain tissue samples were then collected to examine the concentrations of neuron-specific enolase (NSE) and S100- $\beta$ protein. Brain T2WI showed no differences between the groups. $\mathrm{N}$-acetylaspartate/choline (NAA/Cr) ratio measured by MRS showed different degrees of decrease in the sepsis groups, and serum NSE and S100- $\beta$ concentrations were increased compared with the control group. Apoptosis rates were measured in the right hippocampal area, and there were statistically significant differences between the indicated groups and the control group $(\mathrm{p}<0.05)$. The correlation between apoptosis rate and NAA/Cr ratio was closer than that between apoptosis rate and NSE or S100- $\beta$ (-0.925 vs. 0.434 vs. 0.517 , respectively). In conclusion, MRS is a sensitive, non-invasive method to investigate complications of brain injury in septic rats, which may be utilized for the early diagnosis of brain injury caused by sepsis.
\end{abstract}

\section{Introduction}

Sepsis-associated encephalopathy (SAE) is defined as diffuse or multifocal cerebral dysfunction induced by the systemic

Correspondence to: Dr Hongke Zeng, Department of Acute Critical Care Medicine, Guangdong General Hospital, Guangdong Academy of Medical Sciences, 106 Zhongshan Second Road, Guangzhou, Guangdong 510080, P.R. China

E-mail: zenghongke2016@163.com

*Contributed equally

Key words: sepsis, sepsis associated encephalopathy, brain injury, pathology, magnetic resonance spectroscopy, diagnosis response to infection, without clinical or laboratory evidence of direct brain infection (1). SAE is a common complication of brain injury in sepsis, with an incidence of $9-71 \%$ in the intensive care unit (2). In a recent epidemiological multicenter study, the incidence of delirium was $32.3 \%$, in 497 patients with brain injury, including 76 septic patients (3). SAE is not only associated with increased mortality $(4,5)$, but also permanent cognitive dysfunction. Presently, the diagnosis of SAE is a clinical challenge and involves the exclusion of other metabolic and structural etiologies (5). Conventional diagnostic techniques include electroencephalogram (EEG), detection of biomarkers [neuron-specific enolase (NSE) and S100- $\beta$ ] and magnetic resonance imaging (MRI). However, EEG findings are not specific for SAE, and it is often unavailable or unreliable for patients under sedation or with metabolic derangements (1). There are a number of studies suggesting that the diagnosis of SAE does not correlate with the concentration of NSE in serum $(6,7)$. Similar studies indicate that the increase of S100- $\beta$ in septic patients does not correlate with the severity of neurological impairment or with the outcome $(8,9)$. Although MRI can identify structural abnormalities of the brain and other diseases, including SAE, the positive rates of SAE diagnosis are relatively low (10). Thus, MRI is not conducive to early diagnosis and timely intervention of brain injury, because of the deficiencies of conventional diagnostic techniques for SAE.

Magnetic resonance spectroscopy (MRS) is well-established as a non-invasive technique for quantitative analysis of metabolic compounds in living tissues. The degree of brain damage was determined by quantitative detection of metabolic compounds associated with brain injury. N-acetylaspartate (NAA) is a marker of the functional integrity of neuronal mitochondrial metabolism. Its reduction reflects neuronal loss or dysfunction, and is associated with cognitive dysfunction and the degree of brain injury (11). Choline (Cho) is a precursor of the neurotransmitter acetylcholine and of phosphatidylcholine, a major constituent of cell membranes. Increases in Cho reflect increased membrane synthesis and/or increased number of cells, and was shown to be associated with recovery from neuronal injury (12). In spite of its wide application in the diagnosis and assessment of traumatic brain injury, hypoxic ischemic encephalopathy, intracranial 
infections, stroke, cancer, metabolic disorders and other diseases of the brain (13-15), few researchers have explored its role in SAE. Therefore, in the present study, we analyzed the association between the change of MRS and brain injury in the rat model of sepsis, and confirm that MRS technology represents a sensitive method for the early diagnosis of brain injury caused by sepsis.

\section{Materials and methods}

Establishment of the sepsis model. This protocol was approved by the Institutional Animal Care and Use Committee of Guangdong General Hospital (Guangdong, China). Thirty-five adult Sprague-Dawley (SD) rats (weight, 250-300 g) were randomly divided into 4 groups [5 in the control group, and 10 each in the 6, 12 and $24 \mathrm{~h}$ post-lipopolysaccharide (LPS)-injection groups]. Sepsis was induced by a single intraperitoneal administration of LPS (lot. no. B0013K030100; Sigma-Aldrich, St. Louis, MO, USA) at a dose of $30 \mathrm{mg} / \mathrm{kg}$ (16). Instead of LPS, rats in the control group received intraperitoneal injections of normal saline. After injection, all rats were housed in a room with temperature of $22^{\circ} \mathrm{C}$ and humidity of $50 \%$, with a 12-h light/dark cycle and free access to food and water. Clinical symptoms and survival rate were observed at the corresponding time-points $(6,12$ and $24 \mathrm{~h}$ after establishing the sepsis model).

MRI and MRS.MRI and spectroscopy scans were performed in a 7 Tesla horizontal bore magnet (Bruker BioSpec 70/20 USR; Bruker, Karlsruhe, Germany). Rats were deeply anesthetized by a single intraperitoneal administration of $3 \%$ pentobarbital $(2 \mathrm{ml} / \mathrm{kg})$ at 6,12 or $24 \mathrm{~h}$ (control group after $24 \mathrm{~h}$ ) after inducing sepsis. The heads of rats were then fixed in a body restrainer with a tooth-bar and a cone shaped head holder. Body temperature was maintained at $37^{\circ} \mathrm{C}$ with a body heating pad connected to a water heat-exchange system. Respiration was maintained in $800 \mathrm{ml} / \mathrm{min}$ oxygen via a nosecone and monitored with a pressure pad.

Morphological images were acquired using a rapid acquisition relaxation-enhanced (RARE) pulse sequence with T2 weighting (repetition time $=2,000 \mathrm{msec}, \mathrm{TE}=36 \mathrm{msec}$, flip angle $=90^{\circ}$, RARE factor $=8$, average number $=8$, slice thickness $=0.8 \mathrm{~mm}$, number of slices $=16$, field of view $=3.5 \times 3.5 \mathrm{~cm}^{2}$ and $256 \times 256$ matrix).

Spectra were acquired on the region-of-interest of the right hippocampus. The pointed-resolved surface coil spectroscopy pulse sequence was used with the FieldMap with a shimming method $(\mathrm{TR}=2,500 \mathrm{msec}, \mathrm{TE}=20 \mathrm{msec}$, voxel volume $=2 \times 3 \times 3 \mathrm{~mm}^{3}$, average number 512 and acquisition points 2,048). The MRS acquired via measured signal of free induction decay were Fourier-transformed. The spectra were phased, and zero-order baseline was corrected with the software. Spectral windows where changes in signal intensity were identified contained the peaks for NAA (2.02 ppm), Cho (3.21 ppm) and Cr (3.03 ppm). In an effort to limit the sensitivity to phasing artifacts, we computed metabolite ratios for analysis.

Blood sampling and brain tissue preparation. Blood samples and brain tissue were collected immediately after obtaining
MRI and MRS data while rats were maintained under anesthesia. A sternectomy was made to access the heart. A 6-gauge needle (Beyotime, Shanghai, China) was inserted in the left ventricle via the apex for blood sample collection, and samples were then centrifuged at 2,500 x $\mathrm{g}$ for $10 \mathrm{~min}$ at $4^{\circ} \mathrm{C}$. The supernatant was then placed in an Eppendorf tube (Beyotime) and stored at $-70^{\circ} \mathrm{C}$ for detection of NSE and S100- $\beta$. A small opening was cut in the right auricle to allow automatic blood flow. First, rats were rapidly perfused through the left cardiac ventricle with $150 \mathrm{ml}$ normal saline, and then perfusion speed was slowed down until clear liquid flowed out of the right atrium (saline perfusion time was not more than $5 \mathrm{~min}$ ). Then the tissue was fixed by a fixative solution [150 $\mathrm{ml}$ of $4 \%$ paraformaldehyde and $0.1 \%$ glutaraldehyde in $0.1 \mathrm{M}$ phosphate-buffered saline (PBS) (Biosharp, Hefei, China)] until the liver turned yellow and toughened, indicating that perfusion was successful. A craniotomy was performed, in which the right brain tissue was removed for dehydration and paraffin-embedding. Paraffin-embedded tissues were cut coronally into $8-\mu$ m-thick sections for hematoxylin and eosin (H\&E) staining, Nissl staining (lot. no. 201308; Beyotime Institute of Biotechnology, Jiangsu, China), and TUNEL staining (lot. no. 11684817 910; Roche Diagnostics, Basel, Switzerland). Pathological changes of hippocampal tissue were observed by microscopy.

Measurement of serum markers. NSE and S100- $\beta$ levels were analyzed by enzyme-linked immunosorbent assay (ELISA) using commercially available kits (NSE, lot. no. CSB-E07963r and S100- $\beta$, lot. no. CSB-E08066r) (both from Cusabio Biotech Co., Ltd., Wuhan, China) according to the manufacturer's instructions. Microtiter plates with the purified antibody-coating in the solid phase were bound by anti-NSE. Anti-S100- $\beta$ antibody, standards and samples, biotinylated anti-NSE, anti-S100- $\beta$ protein antibody, and HRP-labelled avidin were then added sequentially into micropores. After thorough washing, samples were developed with 3,3',5,5'-tetramethylbenzidine (TMB) substrate. TMB was catalyzed into a blue color via peroxidase and finally changed to yellow after acidification. The absorbance was measured with a microplate reader at $450 \mathrm{~nm}$ [optical density (OD) value], and sample concentrations were calculated according to the standard curve.

Statistical analysis. Statistical analyses were performed using SPSS 17.0 statistical software (SPSS, Inc., Chicago, IL, USA). All statistical assessments were two-sided, and the test statistics and their corresponding P-values were calculated. A value of $\mathrm{p}<0.05$ was considered to indicate a statistically significant difference. Multiple comparisons of enumeration data were performed using the Pearson's Chi-square test or Fisher's exact test. Analysis of variance (ANOVA) was used for multiple comparisons of measurement data where the data were normally distributed. The least significance difference (LSD) test was utilized for the analysis of differences between two groups. The Kruskal-Wallis test was used for multiple comparisons of measurement data where the data were non-normally distributed or exhibited homogeneity of variance. The correlation between variables was analyzed by the Pearson's linear regression test for normal distribution data or Spearman's rankcoefficient test for non-normal distribution data. 


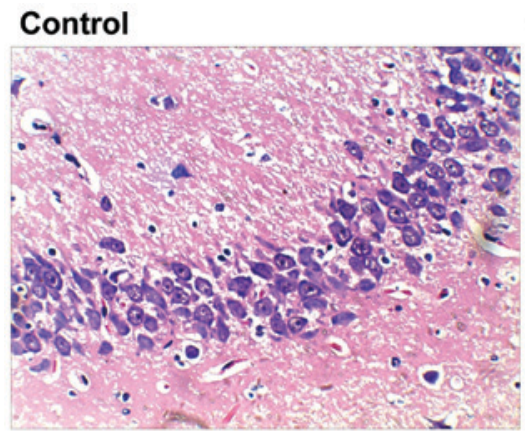

$6 \mathrm{~h}$

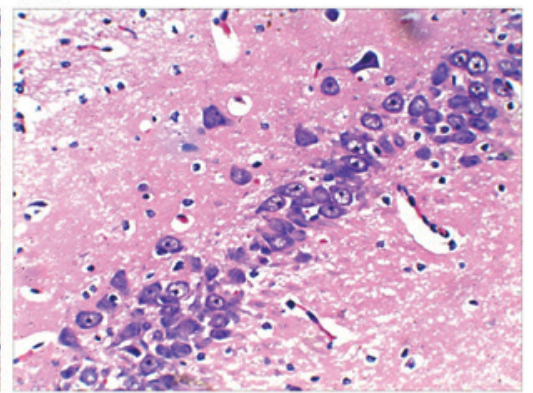

$12 \mathrm{~h}$

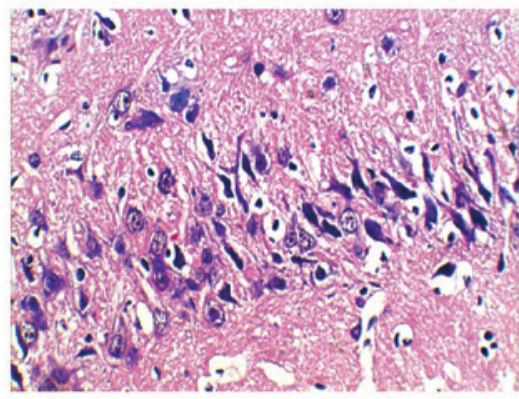

$24 \mathrm{~h}$

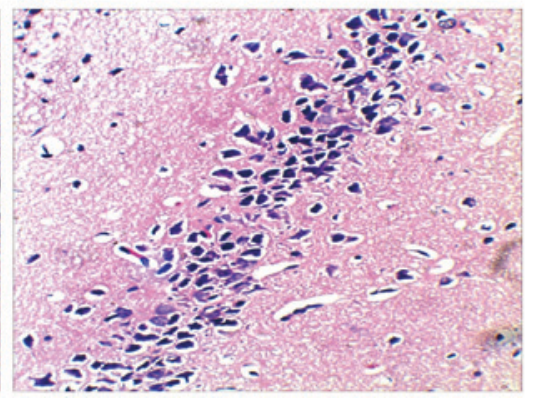

Figure 1. Morphological damage of neurons in right hippocampal areas of septic rats (x400 magnification). The tissue and cells were normal, and there were no pathological changes in the control group. In the $6 \mathrm{~h}$ group, parts of neurons were loosely arranged. Deformation and karyopyknosis were observed in several neurons. In the $24 \mathrm{~h}$ group, the number of neurons were markedly decreased and neurons were loosely arranged. Deformation and karyopyknosis were observed in most neurons. The presentation in the $12 \mathrm{~h}$ group was quite different from that of the $6 \mathrm{~h}$ group, but similar to the $24 \mathrm{~h}$ group.

\section{Results}

Clinical characteristics of the sepsis model. Among the 30 non-control group SD rats, the mortality rates were $30 \%(3 / 10), 40 \%(4 / 10)$, and $40 \%(4 / 10)$ in the 6,12 and $24 \mathrm{~h}$ groups, respectively. All surviving rats showed signs of sepsis, such as malaise, hair-bristle, hemorrhage in the ear and nose, drowsiness and diarrhea. None of the aforementioned symptoms occurred in the control group.

Right hippocampal changes after LPS-induced sepsis. We first performed histopathological analysis of $\mathrm{H} \& \mathrm{E}$ stained sections. After $24 \mathrm{~h}$, hippocampal tissue in rats of the control group showed normal neurons with intact cell structures and nuclear membrane integrity (Fig. 1; control). The $6 \mathrm{~h}$ group exhibited neuronal pyknosis, but no major changes in neural cell morphology or cell structures (Fig. 1; 6 h). Both the 12 and 24 groups had intact histological structure, but showed obvious neuronal pyknosis, swelling of organelles, loss of plasma membrane integrity, and loss of intracellular content, which resulted in dramatically decreased number of neurons and distinctive perimicrovascular edema (Fig. 1; 12 and $24 \mathrm{~h}$ ). Compared with the $6 \mathrm{~h}$ group, the $12 \mathrm{~h}$ group showed increased neural damage, but was similar to that of the $24 \mathrm{~h}$ group.

To quantify cell death, TUNEL staining was used in the right hippocampal area. Examination of the control group revealed no TUNEL-positive cells; whereas, there were a small number of TUNEL-positive cells within the hippocampus of the $6 \mathrm{~h}$ group. In contrast, both the 12 and $24 \mathrm{~h}$ groups showed obvious TUNEL-positive cells (Fig. 2A), and significantly increased formation of apoptotic bodies compared with the control and $6 \mathrm{~h}$ groups $(\mathrm{p}<0.05)$ (Fig. 2B). The apoptotic cells had small cell bodies in the plasma, and tan-color stained nuclei.
A

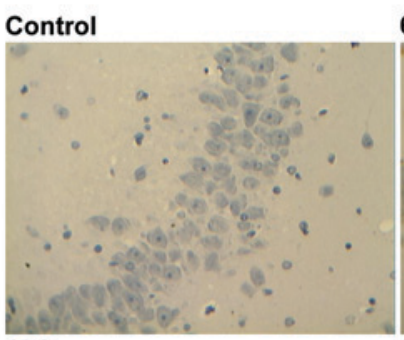

$6 \mathrm{~h}$

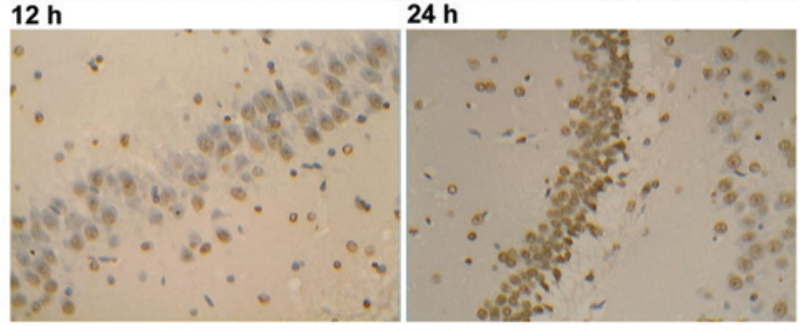

B

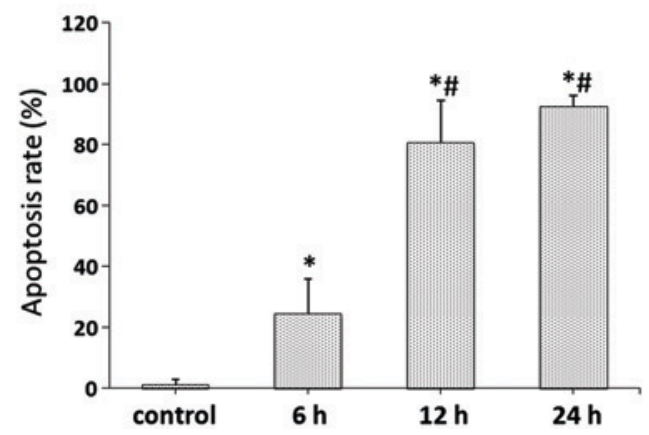

Figure 2. TUNEL staining of rat brain sections at different time-points (x400 magnification). (A) Representative TUNEL-positive signals of the right hippocampal area in control, 6, 12 and $24 \mathrm{~h}$ groups. (B) Quantification of TUNEL results. Data were calculated with the average number of TUNEL-positive signals in five different fields. A statistically significant difference between the indicated group and the control group $\left({ }^{*} \mathrm{p}<0.05\right)$. A statistically significant difference between the indicated group and the $6 \mathrm{~h}$ group $\left({ }^{\#} \mathrm{p}<0.05\right)$. 


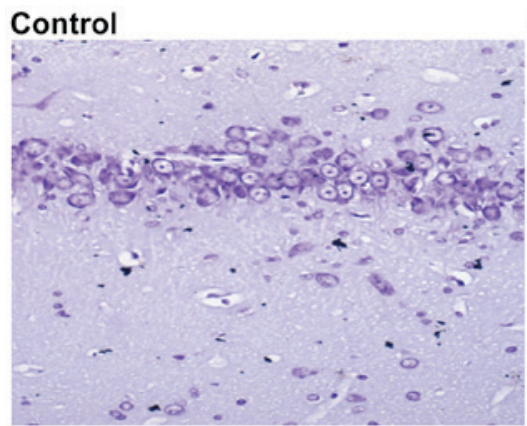

$12 \mathrm{~h}$

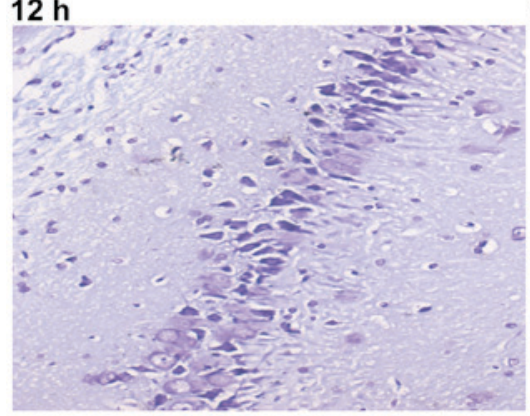

$6 \mathrm{~h}$

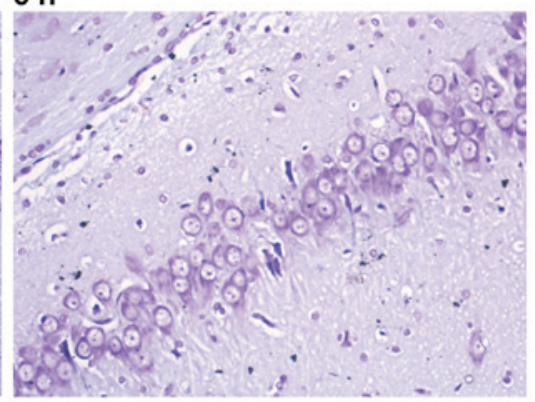

$24 \mathrm{~h}$

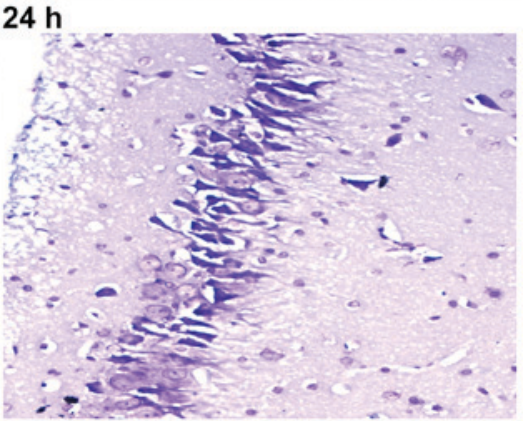

Figure 3. Nissl staining of rat brain sections at different time-points (x400 magnification). Injured neurons were characterized by cytoplasmic shrinkage, nuclear pyknosis and hyperchromasia. Note the abundance of small, darkly stained, dead/dying neurons with remarkable shrinkage in the right hippocampal area. No abnormal Nissl bodies were found in the control group. In the $6 \mathrm{~h}$ group, several cell bodies were pyknotic and deformed. Representative sections from rats after LPS injection show a major dead/dying pattern in the 12 and $24 \mathrm{~h}$ groups. LPS, lipopolysaccharide.
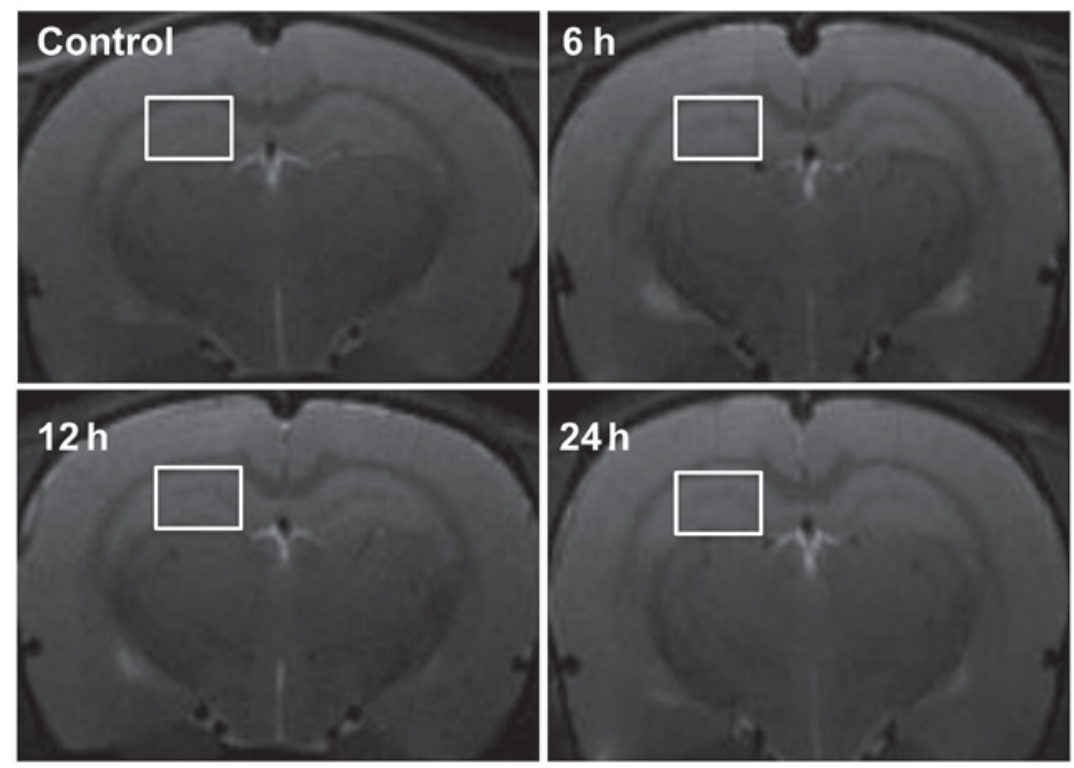

Figure 4. Representative T2WI MRI of the control and LPS-injected groups at the selected time-points. The results showed no significant changes at any of the time-points. White squares on the right hippocampus indicate regions of interest selected for MRS analysis. T2WI, T2-weighted imaging; MRI, magnetic resonance imaging; LPS, lipopolysaccharide; MRS, magnetic resonance spectroscopy.

In addition, they had irregular shapes and were inconsistent in size, showing nuclear condensation and fragmentation of some nuclei, as well as formation of apoptotic bodies.

Nissl staining was then used to evaluate neuron viability at the different time-points. When rats were subjected to sepsis, injured neurons were characterized by cytoplasmic shrinkage, nuclear pyknosis and hyperchromasia. Rats in the control group (Fig. 3, control) did not exhibit any damage or changes in neuronal morphology. Some dead/dying neurons were observed as small, darkly stained, shrunken cells formed in the $6 \mathrm{~h}$ group (Fig. 3; $6 \mathrm{~h}$ ). Compared with the $6 \mathrm{~h}$ group, rats in the 12 and $24 \mathrm{~h}$ groups had more extensive neuronal loss (Fig. 3; 12 and $24 \mathrm{~h}$ ).

$M R I$. To evaluate the diagnostic value of MRI on brain damage of septic rats, we observed the brain tissue of septic rats at 6 , 12 and $24 \mathrm{~h}$ after LPS-injection and in the control group using T2-weighted axial imaging. There were no significant changes 
A
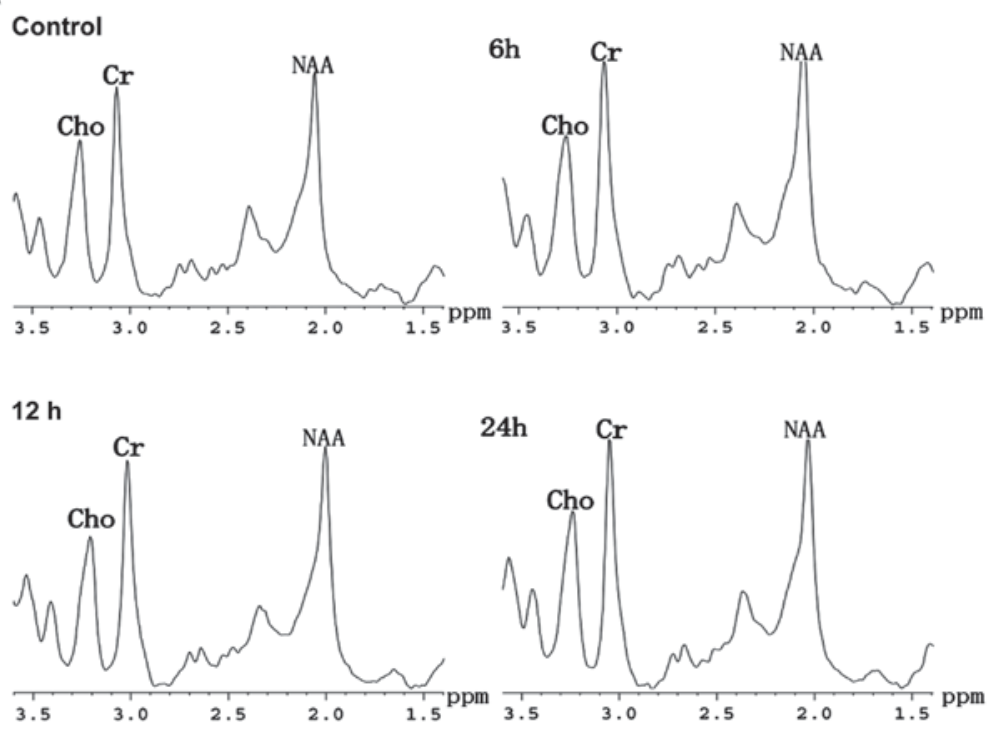

B

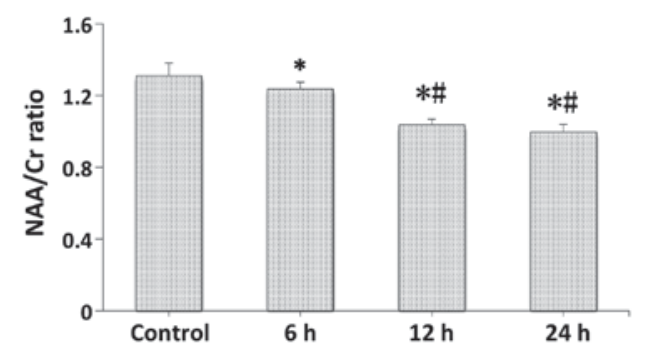

Figure 5. In vivo $1 \mathrm{H}$ MR spectroscopic analysis of brain metabolites. (A) Representative spectra obtained and close-up views of the spectral region encompassing the $\mathrm{Cho}, \mathrm{Cr}$ and NAA peaks for the control, 6,12 and $24 \mathrm{~h}$ groups. The peaks used in the calculation of the ratios are markedly visible at 3.03 and $2.02 \mathrm{ppm}$ for total $\mathrm{Cr}$ and NAA, respectively. (B) Average values \pm SEM of the peak value ratios of NAA to total $\mathrm{Cr}$ in each group. We observed a significant reduction in $\mathrm{NAA} / \mathrm{Cr}$ ratio. A statistically significant difference between the indicated group and the control group ( $\mathrm{p} p<0.05)$. A statistically significant difference between the indicated group and the $6 \mathrm{~h}$ group $\left({ }^{\#} \mathrm{p}<0.05\right)$. MR, magnetic resonance; Cho, choline; NAA, N-acetylaspartate.

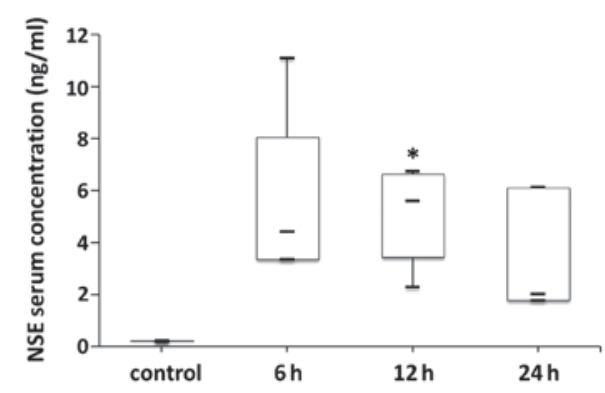

Figure 6. Serum NSE levels in the control group and at different time-points after LPS-induced sepsis. Serum NSE levels in the 6, 12 and $24 \mathrm{~h}$ groups were higher than in the control group. Data are presented as median with interquartile ranges. Median NSE values were 0.22 vs. 4.40 vs. 5.58 vs. $2.01 \mathrm{ng} / \mathrm{ml}$ for the control, 6,12 and $24 \mathrm{~h}$ groups, respectively. ${ }^{*} \mathrm{p}<0.05$. NSE, neuron-specific enolase; LPS, lipopolysaccharide.

at any of the time-points. No high-signal lesions (indicative of cytotoxicity or vasogenic brain edema) or swelling of the lateral ventricles were evident (Fig. 4).

In vivo $1 H$ MR spectra. Proton spectra (Fig. 5A) were acquired in a cubic volume of $18 \mu \mathrm{l}$ in the right hippocampus of the control and LPS-injection groups, and the relative amounts of total NAA and Cr compounds were determined after the identification of their respective peaks (Fig. 5B). Compared

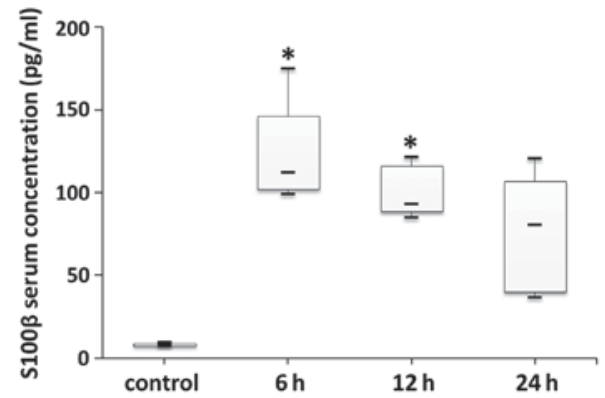

Figure 7. Serum S100- $\beta$ levels in the control group and at different time-points after LPS-induced sepsis. Serum S100- $\beta$ levels in the 6, 12 and 24 h groups were higher than in the control group. Data are presented as median with interquartile ranges. Median S100- $\beta$ values were 7.86 vs. 112.12 vs. 92.78 vs. $80.37 \mathrm{pg} / \mathrm{ml}$ for the control, 6,12 and $24 \mathrm{~h}$ groups, respectively. ${ }^{*} \mathrm{p}<0.05$. LPS, lipopolysaccharide.

with the control group, a strong decrease was seen in the $\mathrm{N}$-acetylaspartate/choline (NAA/Cr) ratio at the different time-points after LPS-injection, and the NAA/Cr ratio in the 12 and $24 \mathrm{~h}$ groups showed a stronger decrease compared with the $6 \mathrm{~h}$ group $(\mathrm{p}<0.05)$.

Measurement of NSE and S100- $\beta$. Both serum NSE and S100- $\beta$ levels at the different time-points after LPS-induced sepsis were higher than in the control group (Figs. 6 and 7). 
Table I. Correlation between two variables.

\begin{tabular}{lrc}
\hline Items & r-values & P-values \\
\hline Apoptosis rate and NAA/Cr ratio & \\
Apoptosis rate and NSE & -0.925 & 0.000 \\
Apoptosis rate and S100- $\beta$ & 0.434 & 0.566 \\
& 0.517 & 0.483 \\
\hline
\end{tabular}

${ }^{a} \mathrm{p}<0.05$ correlation between two variables. NAA, N-acetylaspartate; NSE, neuron-specific enolase.

There were statistically significant differences in both serum NSE and S100- $\beta$ levels between the control and $6 \mathrm{~h}$ groups $(p<0.05)$. In addition, there was a statistically significant difference in serum S100- $\beta$ levels between the control and $12 \mathrm{~h}$ groups $(\mathrm{p}<0.05)$. However, there were no statistically significant differences in serum NSE or S100- $\beta$ levels between the $6,12 \mathrm{~h}$ or $24 \mathrm{~h}$ groups $(\mathrm{p}>0.05)$.

Correlation analysis between two variables. The correlation between apoptosis rate and the NAA/Cr ratio was stronger than that between apoptosis rate and NSE or S100- $\beta(-0.925$ vs. 0.434 vs. 0.517 , respectively) (Table I).

\section{Discussion}

Although the pathogenesis of SAE is still unclear, early diagnosis and treatment are critical to reduce mortality and disability from brain damage in septic patients. However, the methods of assessing brain injury such as EEG (1), T2WI (10), NSE $(6,7)$ and S100- $\beta(8,9)$ have limitations. Brain MRS is a non-invasive method to measure the concentrations of biochemical compounds in MRI-specified areas of cerebral tissue (17). This technique offers the ability to acquire in vivo data on biochemical compounds with negligible influence on the disease process, and allows the detection of even small deviations of normal brain function in humans and animal models (18). Here, we report the implementation of brain T2-weighted imaging (T2WI), proton spectroscopy and serum markers including NSE and S100- $\beta$, in LPS-induced septic rats. The results were compared with histopathological analysis. Overall, compared with T2WI and serum markers, the results obtained by MRS were closer to the histopathological results of neuronal damage. The results of our study contribute valuable and novel information for the early diagnosis of brain injury caused by sepsis.

The histopathology presented herein indicates that brain damage can be detected by any kind of dye used in the present study in the early (up to $6 \mathrm{~h}$ ) period of LPS-induced sepsis in rats. In addition, compared with the control group, the damage in the 12 and $24 \mathrm{~h}$ groups was most severe. Messaris et al (19) confirmed the presence of apoptosis in the hippocampus, and this process began $6 \mathrm{~h}$ after initiation of experimental sepsis and increased in the late phases of sepsis. The importance of apoptosis in the immunologic and pathological mechanisms of sepsis has been recognized (20). However, in 1998, Mouihate and Pittman (21) found no evidence of apoptosis in the hippocampus and other brain regions of rats $5.5 \mathrm{~h}$ and
5 days after intraperitoneal injection of LPS. The reasons for the discrepancy in these results remain unclear. It may be related to several factors, such as differences in species or age of rats, different amounts of LPS, or the time-points of observation.

In the present study, little to no changes in T2WI could be detected either in the early $(6 \mathrm{~h})$ or late $(24 \mathrm{~h})$ phase of LPS-induced sepsis in rats. Although MRI can be utilized for the exclusion of cerebral dysfunction from other cerebrovascular conditions, it is not a specific test for SAE (1). In fact, we report that rats began to have symptoms of brain disorder in the early period of sepsis, such as mental fatigue and drowsiness, and pathological analysis also indicated brain damage, but the results from MRI showed normal brain. However, it was recently revealed that accumulation of vasogenic edematic fluid at the base of the brain was observed by T2WI at 6 and $24 \mathrm{~h}$ after cecal ligation and puncture (CLP)-induced sepsis (18). This apparent discrepancy may have been because of the differences in the experimental models. The inflammatory processes mediated by CLP are more intensely stimulated by polymicrobial sepsis.

MRS measurements indicated alterations in the NAA/Cr ratio. These were clearly apparent in the hippocampus of septic rats as early as $6 \mathrm{~h}$ after LPS-induction. Moreover, the decrease in NAA/Cr ratio in the hippocampus was more significant at 12 and $24 \mathrm{~h}$. Similar findings were reported by Bozza et al at 6 and $24 \mathrm{~h}$ after experimental sepsis (18). However, our study confirms the presence of apoptosis by TUNEL staining, and the results showed that the decrease in NAA/Cr ratio was consistent with the apoptosis rate. This indicates that the induction of apoptosis in the hippocampus is an important component of the pathogenesis of brain dysfunction in sepsis, as confirmed earlier by Sharshar et al (22). Taken together, our findings indicated that neuronal damage was accompanied by decreased NAA levels in septic rats (23-25).

Some researchers believed that NSE and S100- $\beta$ may be biomarkers of brain damage, and can be used as a testing index for sepsis in clinic $(1,26)$. Here, we compared the levels of NSE and S100- $\beta$ in the 6, 12 and $24 \mathrm{~h}$, and control group. The results showed that although the protein concentrations of NSE and S100- $\beta$ were different in the serum of each group, the statistical differences were inconsistent with the pathological analyses. The results of our study are consistent with those of others that showed no correlation between serum concentrations of NSE and S100- $\beta$ and the severity of SAE $(7,8)$. Specifically, they asserted that the rise of S100- $\beta$ concentration may originate from outside the brain (the amount of S100- $\beta$ in chondrocytes and adipose cells is $1 / 4$ that of the brain).

Despite the novelty of our findings, we acknowledge some limitations to our study. In this study, we did not monitor blood pressure during the MRS procedure. Therefore, we cannot rule out the influence of hemodynamics on the complications of brain damage after LPS-induced sepsis. However, it was shown that rats subjected to CLP (with a mortality rate of $43 \%$ ) did not exhibit alterations in mean whole or local brain blood flow, and the authors concluded that brain dysfunction is not a consequence of changes of cerebral blood flow during severe sepsis (25). Moreover, there was an absence of EEG monitoring because the MRS procedure requires a better condition. These areas will be investigated in our future studies. 
Taken together, relative to T2WI and serum biomarkers, our observations indicate that MRS is a suitable non-invasive method to investigate the complications of brain damage in septic rats. Significant quantitative changes were detected in the metabolic profiles of septic animals, which were consistent with the apoptosis rate, and may ultimately result in improvements of the diagnostic accuracy and severity assessment of SAE in patients.

\section{Acknowledgements}

This study was supported by the Medical Science and Technology Foundation of Guangdong Province (B2013015), and the Science and Technology Foundation of Guangdong Province (2013B022000081). The authors are thankful for the support received from Dr Peter Hou and Dr Kun Xiao during the process of manuscript development.

\section{References}

1. Cotena S and Piazza O: Sepsis-associated encephalopathy. Trans Med UniSa 2: 20-27, 2012.

2. Young GB, Bolton CF, Austin TW, Archibald YM, Gonder J and Wells GA: The encephalopathy associated with septic illness. Clin Invest Med 13: 297-304, 1990.

3. Salluh JI, Soares M, Teles JM, Ceraso D, Raimondi N, Nava VS, Blasquez P, Ugarte S, Ibanez-Guzman C, Centeno JV, et al; Delirium Epidemiology in Critical Care Study Group: Delirium epidemiology in critical care (DECCA): an international study. Crit Care 14: R210, 2010

4. Zampieri FG, Park M, Machado FS and Azevedo LC: Sepsis-associated encephalopathy: not just delirium. Clinics (Sao Paulo) 66: 1825-1831, 2011.

5. Pytel P and Alexander JJ: Pathogenesis of septic encephalopathy. Curr Opin Neurol 22: 283-287, 2009.

6. Rodríguez-Núñez A, Cid E, Rodríguez-García J, Camiña F, Rodríguez-Segade S and Castro-Gago M: Concentrations of nucleotides, nucleosides, purine bases, oxypurines, uric acid, and neuron-specific enolase in the cerebrospinal fluid of children with sepsis. J Child Neurol 16: 704-706, 2001.

7. van den Boogaard M, Ramakers BP, van Alfen N, van der Werf SP, Fick WF, Hoedemaekers CW, Verbeek MM, Schoonhoven L, van der Hoeven JG and Pickkers P: Endotoxemia-induced inflammation and the effect on the human brain. Crit Care 14 R81, 2010.

8. Piazza O, Russo E, Cotena S, Esposito G and Tufano R: Elevated S100B levels do not correlate with the severity of encephalopathy during sepsis. Br J Anaesth 99: 518-521, 2007.

9. Heizmann CW: S100B protein in clinical diagnostics: assay specificity. Clin Chem 50: 249-251, 2004.

10. Piazza O, Cotena S, De Robertis E, Caranci F and Tufano R: Sepsis associated encephalopathy studied by MRI and cerebral spinal fluid S100B measurement. Neurochem Res 34: 1289-1292, 2009.
11. Lim MK, Suh CH, Kim HJ, Cho YK, Choi SH, Kang JH, Park W and Lee JH: Systemic lupus erythematosus: brain MR imaging and single-voxel hydrogen 1 MR spectroscopy. Radiology 217: 43-49, 2000.

12. Castillo M, Kwock L and Mukherji SK: Clinical applications of proton MR spectroscopy. AJNR Am J Neuroradiol 17: $1-15,1996$.

13. Cohen BA, Inglese M, Rusinek H, Babb JS, Grossman RI and Gonen O: Proton MR spectroscopy and MRI-volumetry in mild traumatic brain injury. AJNR Am J Neuroradiol 28: 907-913, 2007.

14. Inder TE and Volpe JJ: Mechanisms of perinatal brain injury. Semin Neonatol 5: 3-16, 2000.

15. Mishra AM, Gupta RK, Jaggi RS, Reddy JS, Jha DK, Husain N, Prasad KN, Behari S and Husain M: Role of diffusion-weighted imaging and in vivo proton magnetic resonance spectroscopy in the differential diagnosis of ring-enhancing intracranial cystic mass lesions. J Comput Assist Tomogr 28: 540-547, 2004.

16. Lin LC, Chen YY, Lee WT, Chen HL and Yang RC: Heat shock pretreatment attenuates sepsis-associated encephalopathy in LPS-induced septic rats. Brain Dev 32: 371-377, 2010.

17. Van Zijl PC and Barker PB: Magnetic resonance spectroscopy and spectroscopic imaging for the study of brain metabolism. Ann N Y Acad Sci 820: 75-96, 1997.

18. Bozza FA, Garteiser P, Oliveira MF, Doblas S, Cranford R, Saunders D, Jones I, Towner RA and Castro-Faria-Neto HC: Sepsis-associated encephalopathy: a magnetic resonance imaging and spectroscopy study. J Cereb Blood Flow Metab 30: 440-448, 2010

19. Messaris E, Memos N, Chatzigianni E, Konstadoulakis MM, Menenakos E, Katsaragak is S, Voumvourakis $\mathrm{C}$ and Androulakis G: Time-dependent mitochondrial-mediated programmed neuronal cell death prolongs survival in sepsis. Crit Care Med 32: 1764-1770, 2004

20. Ayala A, Perl M, Venet F, Lomas-Neira J, Swan R and Chung CS: Apoptosis in sepsis: mechanisms, clinical impact and potential therapeutic targets. Curr Pharm Des 14: 1853-1859, 2008.

21. Mouihate A and Pittman QJ: Lipopolysaccharide-induced fever is dissociated from apoptotic cell death in the rat brain. Brain Res 805: 95-103, 1998.

22. Sharshar T, Gray F, Lorin de la Grandmaison G, Hopkinson NS, Ross E, Dorandeu A, Orlikowski D, Raphael JC, Gajdos P and Annane D: Apoptosis of neurons in cardiovascular autonomic centres triggered by inducible nitric oxide synthase after death from septic shock. Lancet 362: 1799-1805, 2003.

23. Davies DC: Blood-brain barrier breakdown in septic encephalopathy and brain tumours. J Anat 200: 639-646, 2002.

24. Orihuela CJ, Fillon S, Smith-Sielicki SH, El Kasmi KC, Gao G, Soulis K, Patil A, Murray PJ and Tuomanen EI: Cell wall-mediated neuronal damage in early sepsis. Infect Immun 74: 3783-3789, 2006

25. Papadopoulos MC, Lamb FJ, Moss RF, Davies DC, Tighe D and Bennett ED: Faecal peritonitis causes oedema and neuronal injury in pig cerebral cortex. Clin Sci (Lond) 96: 461-466, 1999.

26. Undén J, Christensson B, Bellner J, Alling C and Romner B: Serum S100B levels in patients with cerebral and extracerebral infectious disease. Scand J Infect Dis 36: 10-13, 2004. 